\title{
Greater Spending Associated with Improved Survival for Some Cancers in OCM-Defined Episodes
}

\author{
James Baumgardner, PhD; Ahva Shahabi, PhD; Mark Linthicum, MPP; \\ Seanna Vine, MPH; Christopher Zacker, RPh, PhD; and Darius Lakdawalla, PhD
}

\begin{abstract}
BACKGROUND: Previous research finds significant variation in spending and utilization across regions, with little evidence of differences in outcomes. While such findings have been interpreted as evidence that spending can be reduced without compromising patient outcomes, the link between spending variation and outcomes remains a critical question.

OBJECTIVE: To use evidence from geographic variations in spending and an individual-level survival analysis to test whether spending within oncology care episodes is associated with survival, where episodes are defined as in the Center for Medicare and Medicaid Innovation's Oncology Care Model (OCM).
\end{abstract}

METHODS: In this retrospective cohort analysis, patient data from the Surveillance, Epidemiology and End Results Medicare (SEER-Medicare) database for 2007-2013 were linked to hospital referral regions (HRRs) using ZIP codes. Patients in the SEER program are a part of selected population-based cancer registries throughout the United States whose records are linked to Medicare enrollment and claims data (93\% of elderly registry patients were successfully linked to Medicare data). Episodes of cancer care were defined as in the OCM: 6 months following a triggering chemotherapy claim. We analyzed episodes of care for 5 tumor types: advanced breast cancer (BC), non-small cell lung cancer (NSCLC), renal cell carcinoma (RCC), multiple myeloma (MM), and chronic myeloid leukemia (CML). We removed the effects of differentials in Medicare payment rates, which were mostly geographic. Regression analysis was then used to calculate standardized spending levels for each HRR, that is, spending adjusted for differences in patient and episode characteristics. To examine the effect of spending during $0 \mathrm{CM}$-defined episodes on individual-level survival, we used Cox regression with patient characteristics and standardized HRR spending per episode as covariates. To address concerns that may arise from multiple comparisons across the 5 tumor types, we used the Benjamini-Hochberg procedure to control the false discovery rate.

RESULTS: Our analysis showed significant differences in standardized spending across HRRs. Compared with spending at the 20th percentile episode, spending at the 80th percentile ranged from $25 \%$ higher $(\$ 57,392$ vs. $\$ 45,995$ for MM) to $47 \%$ higher $(\$ 36,920$ vs. $\$ 24,127$ for RCC), indicating practice style variation across regions. The hazard of dying for patients with NSCLC and MM statistically significantly decreased by $7 \%(\mathrm{HR}=0.93$, $P=0.006)$ and $13 \%(H R=0.87, P=0.019)$, respectively, for a $\$ 10,000$ increase in standardized spending (in 2013 U.S. dollars). For the 3 other cancers, spending effects were not statistically significant. After using the Benjamini-Hochberg procedure with a $5 \%$ false discovery rate, the effects of increased spending on improved survival for NSCLC and MM remained statistically significant.

CONCLUSIONS: The association we found between spending and survival suggests caution may be warranted for physicians, pharmacists, other health care professionals, and policymakers involved in efforts to reduce across-the-board spending within OCM-defined episodes for at least 2 of the 5 cancers studied.

J Manag Care Spec Pharm. 2018;24(6):504-13

Copyright $\odot 2018$, Academy of Managed Care Pharmacy. All rights reserved.

\section{What is already known about this subject}

Some innovative payment models such as the Center for Medicare and Medicaid Innovation's Oncology Care Model (OCM) aim to reduce overall costs for cancer care while improving the quality of care.

Past research on geographic variations in health care generally suggests that spending variation is attributable to local area differences in providers' practice styles, with conflicting results as to whether greater spending is associated with better or worse outcomes for patients

\section{What this study adds}

Significant variation was documented across regions in spending per episode for 5 cancer types after adjustment for differences in patient and episode characteristics and in Medicare payment rates. The association between spending and survival differed across cancer types; for example, the hazard of dying for patients with non-small cell lung cancer (NSCLC) and multiple myeloma (MM) decreased by $7 \%(\mathrm{HR}=0.93, P=0.006)$ and $13 \% \quad(\mathrm{HR}=0.87$, $P=0.019$ ), respectively, for a $\$ 10,000$ increase in standardized spending, whereas spending effects were not statistically significant for the other 3 cancer types studied.

Study results suggest that health care providers should consider exercising caution when attempting to reduce spending in response to new payment models, especially in cases of NSCLC and MM

$\mathrm{P}$ ayers are experimenting with alternative payment models (APMs) that include incentives to reduce spending on the treatment of cancer patients. ${ }^{1}$ For example, the Center for Medicare and Medicaid Innovation initiated a demonstration project called the Oncology Care Model (OCM) in 2016. 2-4 Shared savings models such as the OCM and other APMs seek to reduce spending by moving away from payment for volume of services provided and toward payment for value or better patient outcomes, with the assumption that significant unnecessary spending currently exists.

In the case of the OCM, an oncology practice receives a performance-based payment dependent on a practice's performance on several measures of quality and the extent to which spending per episode is kept below a target set to yield savings to the Medicare program. The OCM defines an episode as the 
6-month period initiated by outpatient chemotherapy, either infused or oral. ${ }^{3}$ Because the OCM creates incentives to reduce spending in episodes so defined, a crucial question is whether reduced spending in those episodes would adversely affect patient outcomes despite the potential effect of the quality improvement incentives.

The general spirit of findings in the well-established literature on geographic variations in health care has been of significant variation in spending across regions, often attributed to differences in the practice styles of physicians in different areas, with little and conflicting evidence of differences in outcomes. ${ }^{5-7}$ Such findings have been interpreted as evidence that spending can be reduced without compromising patient outcomes, if, for instance, the higher spenders in the health care system were incentivized to behave like the lower spenders. ${ }^{6}$

In this analysis, for 5 cancer types we measured the geographic variation in spending, which may be indicative of inefficient spending, and the association between spending and survival, which may indicate potential consequences of general spending reductions on outcomes unless they are offset by quality improvement incentives. We measured spending per OCM-defined episode, since that is the unit over which practices are incentivized to reduce spending within the OCM. Our analysis can inform policymakers and health care professionals of potential concerns in pursuing APMs such as the OCM, which imbed incentives for general spending reductions along with incentives to improve quality of care. Our analysis made no attempt to evaluate the effect of the OCM methodology as a whole or, more specifically, the effects of its quality metrics.

\section{Methods}

We used a geographic variations approach to (a) assess the amount of interregional variation in spending on cancer treatment, which has typically been interpreted as evidence of differences in physicians' practice styles across regions, and (b) examine the association between spending and patient survival. An advantage of our approach is that we estimated survival functions using individual-level data, which allowed us to control for individual characteristics and to make use of complete and incomplete survival spells with the Cox survival model.

Our approach also avoided the spurious correlation that would exist in a survival analysis conducted entirely at the individual level. In particular, if one simply regressed individual survival on patient-level spending and other covariates, unobserved differences in severity would likely cause higher spending to be associated with lower survival because treatment of patients with poorer prognoses tends to be more resource intensive. This approach would lead to the incorrect inference that higher spending had harmed the patients, when instead, their earlier deaths owed to the greater severity of their illnesses at baseline.
To avoid confounding by a patient's unmeasured disease severity, we regressed patient survival on the local region's spending per episode on a standardized patient and on other individual-level covariates. In essence, we use the local area's level of spending on a standardized patient as a means of measuring variation in spending that is unrelated to the individual's disease severity. We analyzed whether patients who live in areas where practice styles incorporate higher spending have significantly better survival. Our estimate removed the effects of observable differences in patients and episodes and of payment rate differentials across areas.

\section{Data Source}

Our study used data from the Surveillance, Epidemiology and End Results-Medicare (SEER-Medicare) database for patients who received chemotherapy treatment in the 2007-2013 period. The SEER-Medicare database links cancer registry data with longitudinal Medicare claims for Parts A, B, and D. We also used data from 2006 in order to create the Charlson index of comorbidities based on diagnoses observed in the 12 months before a cancer treatment episode. The data allowed us to follow patients during the study period from Medicare enrollment to death or December 31, 2013, which was the most recent date of availability for the SEER-Medicare files at the time we conducted the analysis. The Medicare data also include date of death, obtained from the Social Security Administration, which allows for reliable measurement of survival duration.

\section{Cancer Types Included}

We identified patients diagnosed with 1 of the following tumor types as the primary cancer: advanced breast cancer (BC), nonsmall cell lung cancer (NSCLC), renal cell carcinoma (RCC), multiple myeloma (MM), and chronic myeloid leukemia (CML). These tumor types were chosen to provide variety in terms of incidence and prevalence in the Medicare population, the innovation and resource mix used in treatment, and a mix of solid and hematological cancers. In the case of BC, we focused on advanced stage (stages 3 and 4) diagnoses because the longer survival times of earlier stage patients would have resulted in few deaths occurring within our data window, leaving little variation to measure. Our patient cohort included patients newly diagnosed with 1 of the 5 selected tumors between January 1, 2007, and December 31, 2011. Recoded International Classification of Diseases for Oncology, Third Revision (ICD-O-3) codes were used to identify patients with these 5 tumor types (BC: 26000, NSCLC: 22030, RCC: 29020, MM: 34000, and CML: 35022). Details on additional histologic codes used appear in Appendix A (available in online article).

We restricted the study cohort to patients enrolled in Parts A, B, and D throughout their time in our data window. Since the OCM excludes patients enrolled in Medicare Advantage, those with end-stage renal disease, and those with a primary 
payer other than Medicare, we excluded those groups. We restricted the sample to patients treated with at least 1 OCMeligible chemotherapy drug in an outpatient setting (including Part D chemotherapy claims). This restriction allowed us to define an episode in the same way as the OCM.

\section{Episode Definition}

We followed the OCM definition of an episode of care: 6 months (180 days) triggered by initiation of outpatient chemotherapy, either infused/injected or oral. If a patient continued or resumed outpatient chemotherapy after the end of the episode, then a new 6-month episode began. This was continued through the end of the data or their death; a final episode could be less than 6 months, as in the OCM.

\section{Standardization for Medicare Payment Rate Differentials}

Using the Medicare claims, we calculated the base cost for each claim. In general, the base cost was the sum of the payment amount from Medicare and deductible and coinsurance amounts. Each claim payment was standardized to remove the effect of differentials across geographic areas (and in the case of inpatient hospital payments, across hospitals in the same region) in Medicare payment rates for the same service. This analytic step eliminated variation in spending due to pricing or payment rate differences. Because our spending data covered several years, we also corrected for changes in payment rates over time (including the sequester), with a normalization to (presequester) 2013 U.S. dollars. Details on our standardization methods appear in Appendix A. As a result of our standardization, when we measured higher spending, it reflected either greater utilization or greater use of goods and services having higher average costs.

\section{Survival}

We measured survival as duration between diagnosis date and date of death and used a Cox survival model (see the "Statistical Analysis" section) for estimating the effects of covariates. For patients who did not die during the study period, we treated their survival duration as right-censored. The Cox model used information from both censored and noncensored cases in estimating the parameters of the survival function. Patients who were diagnosed at the beginning of our sample frame had the potential to remain alive and in the sample for up to 7 years (January 2007-December 2013).

\section{Defining the Geographic Market}

Hospital referral regions (HRRs) developed by the Dartmouth Atlas of Health Care were used to define regional health care markets. ${ }^{8}$ The HRRs have been widely used to define geographic markets for use in studies of the variation in use of medical services. Medicare beneficiaries receive over $80 \%$ of their care within their HRRs. ${ }^{9}$ Patient ZIP code of residence, obtained through an additional application process with SEERMedicare, was used to map patients to their respective HRRs.

\section{Spending and Covariates Used in Regression Analysis}

We calculated spending per OCM-defined episode by first identifying use of outpatient chemotherapy and then applying the OCM definition of an episode. We aggregated all claims within each episode to calculate spending per episode. We included all spending on Parts A, B, and D services.

For the spending regressions, covariates included age, sex, eligibility for Medicaid, disability, Charlson Comorbidity Index (CCI) score, disease stage at diagnosis, number of previous episodes for the patient, death during the episode, and the geographic region of residence. The survival analysis included indicator variables for race and ethnicity, as well as other patient characteristics.

\section{Statistical Analysis}

Step 1: Estimation of Spending per Episode Regressions. A generalized linear model with a gamma distribution and log-link function was used to regress spending per episode on characteristics of the patient, other control variables, and an indicator for the patient's geographic region (HRR). To account for the correlation of episodes for each patient, we clustered at the patient level, which allowed for estimation of cluster robust standard errors. Separate regression models were run for each cancer type.

Step 2: Calculation of Standardized Spending per Episode for Each Region. The regression results from step 1 were used to calculate a value of spending per episode for each HRR for a patient with a standardized set of characteristics, which were defined using mean values of the covariates over the entire sample for each respective cancer. The distribution of standardized spending per episode across HRRs can be interpreted as reflecting practice style differences across the providers in different regions.

Step 3: Estimation of Individual Survival Regressions. To examine the effect of spending on survival, we used a Cox regression model with individual patients as the unit of observation. Regressors included patient characteristics, and the key variable of interest was the standardized spending per episode for the patient's HRR. Because the latter variable was calculated using the estimated coefficients from the spending regression, bootstrap standard errors were calculated where the spending regression, calculation of HRR standardized spending, and the Cox regression were estimated together in each round of the bootstrap. The Schoenfeld's residuals test was used to test the proportional hazards assumption of the Cox model. Because this analysis was performed on all 5 cancers to determine if spending affects survival, concern about multiple comparisons exists. Therefore, the Benjamini-Hochberg procedure ${ }^{10}$ to 
TABLE 1 Characteristics of Adult Cancer Patients Diagnosed 2007-2011 and Who Received Treatment 2007-2013 Within 6-Month Episodes of Care as Defined by the OCM

\begin{tabular}{|c|c|c|c|c|c|}
\hline \multirow[b]{2}{*}{ Level } & \multicolumn{5}{|c|}{ Cancer Type } \\
\hline & BC & NSCLC & RCC & MM & CML \\
\hline \multicolumn{6}{|l|}{ Disease } \\
\hline Total number of 6-month episodes & 16,039 & 25,697 & 2,711 & 7,246 & 2,163 \\
\hline Total number of patients & 3,593 & 14,380 & 1,224 & 2,218 & 466 \\
\hline Number of included HRRs with $\geq 20$ episodes in each HRR & 102 & 112 & 69 & 91 & 64 \\
\hline \multicolumn{6}{|l|}{ Patient } \\
\hline Mean age at diagnosis (SD) & $73.0 \quad(10.6)$ & $72.4 \quad(8.10)$ & $(8.8)$ & $74.7 \quad(8.3)$ & $72.6 \quad(11.2)$ \\
\hline Mean CCI score at first episode (SD) & $1.0 \quad(1.3)$ & $(1.4)$ & $(1.7)$ & (1.6) & $(1.7)$ \\
\hline Mean months of follow-up time (SD) & $35.9 \quad(21.3)$ & $19.7 \quad(18.3)$ & $31.8 \quad(22.6)$ & $32.2 \quad(20.8)$ & $34.4 \quad(22.6)$ \\
\hline Median survival, months ${ }^{\mathrm{a}}$ & Not reached & 20 & 62 & 70 & Not reached \\
\hline Percentage male (n) & Excluded & $49.8(7,157)$ & $55.6 \quad(681)$ & $45.9 \quad(1,017)$ & $50.9 \quad(237)$ \\
\hline \multicolumn{6}{|l|}{ Race/ethnicity } \\
\hline Percentage white (n) & $80.4(2,887)$ & $79.5(11,427)$ & $77.3 \quad(946)$ & $73.4(1,627)$ & 79.6 \\
\hline Percentage black (n) & $12.6 \quad(452)$ & $10.5(1,511)$ & $8.9 \quad(109)$ & $14.8 \quad(329)$ & $(42)$ \\
\hline Percentage other (n) & $7.0 \quad(254)$ & $10.0(1,442)$ & $13.8 \quad(169)$ & $11.8 \quad(262)$ & 11.4 \\
\hline \multicolumn{6}{|l|}{ Stage at diagnosis ${ }^{b}$} \\
\hline Percentage stage l (n) & NA & $17.1(2,299)$ & $(426)$ & NA & NA \\
\hline Percentage stage 2 (n) & NA & $6.3 \quad(855)$ & $(70)$ & NA & NA \\
\hline Percentage stage 3 (n) & $65.2(2,342)$ & $32.0(4,309)$ & $(176)$ & NA & NA \\
\hline Percentage stage $4(n)$ & $34.8(1,251)$ & $44.6(6,017)$ & $41.9 \quad(484)$ & NA & NA \\
\hline Percentage entitled to Medicare through disability (n) & $23.3 \quad(836)$ & $24.2(3,487)$ & $21.7 \quad(266)$ & $15.6 \quad(346)$ & $26.6 \quad(124)$ \\
\hline Percentage Medicaid eligible (n) & $40.8(1,467)$ & $38.7(5,565)$ & $(518)$ & $33.2 \quad(737)$ & $39.9 \quad(186)$ \\
\hline \multicolumn{6}{|l|}{ Episode } \\
\hline Mean number of previous episodes (SD) & $(2.5)$ & $(1.5)$ & $(1.9)$ & $(2.1)$ & $(2.8)$ \\
\hline Mean unadjusted total spending per episode in USD (SD)c & $\begin{array}{c}20,887.3 \\
(22,600.6)\end{array}$ & $\begin{array}{c}39,544.1 \\
(27,435.3)\end{array}$ & $\begin{array}{c}33,553.3 \\
(27,186.2) \\
\end{array}$ & $\begin{array}{c}52,489.3 \\
(29,452.9) \\
\end{array}$ & $\begin{array}{c}40,452.1 \\
(24,704.3) \\
\end{array}$ \\
\hline Percentage of episodes ended due to death (n) & $7.7 \quad(1,242)$ & $32.2(8,273)$ & $19.8 \quad(538)$ & $11.5 \quad(830)$ & $7.5 \quad(163)$ \\
\hline
\end{tabular}

a Median survival "not reached" indicates that more than $50 \%$ of the study population was still alive by the end of the study follow-up (median survival could not be calculated).

batients with missing stage at diagnosis: NSCLC, $n=900$ ( 6\%) and RCC, $n=68(\sim 5.5 \%)$.

${ }^{c}$ Mean episode spending was adjusted for Medicare payment rate differentials but not for patient characteristics.

$B C=$ advanced breast cancer (stages 3 and 4); CCI = Charlson Comorbidity Index; $C M L=$ chronic myeloid leukemia; HRR=hospital referral region; $M M=$ multiple myeloma; NA = not available; NSCLC=non-small cell lung cancer; OCM= Oncology Care Model; RCC = renal cell carcinoma; SD=standard deviation; USD=U.S. dollars.

control the false discovery rate (FDR) was used to assess statistical significance among our 5 results with an FDR of 5\% (i.e., among significant results, $5 \%$ are acceptable as false positives).

\section{Results}

Summary statistics appear in Table 1. Because of differences in disease prevalence, our sample contained more NSCLC and advanced BC episodes and patients and relatively fewer RCC and CML episodes and patients. HRRs were included in the analysis for a particular cancer if there were at least 20 episodes from patients in the HRR. Hence, our number of included HRRs ranged from 64 for CML to 112 for NSCLC. Average age at diagnosis was similar across our cancer types, ranging from 72.4 for NSCLC to 74.7 for MM. NSCLC was shown to have the lowest median survival (20 months) in a Kaplan-Meier analysis, with the longest survival for advanced BC and CML. Average total spending per episode (unadjusted for differences in patient characteristics) ranged from just under $\$ 21,000$ for advanced BC to over $\$ 52,000$ for MM.

Although some qualitative differences exist across the cancer types, the effects of covariates on spending per episode were similar when we controlled for regional variations in practice styles (Table 2). Spending per episode decreased with age for all 5 cancer types when evaluated at mean age (all $P<0.001$ ). More advanced disease stages at diagnosis were associated with statistically significant increases in spending when compared with stage 1 , ranging from $5.6 \%$ for stage 2 NSCLC (coefficient $=0.056, P=0.05$ ) to $67.9 \%$ for stage 4 RCC (coefficient $=0.679, \quad P<0.001$ ), although spending did not increase monotonically with stage in all cancers. Similarly, comorbidities (CCI) were associated with statistically significant increases in spending across cancers at mean CCI except for MM, where the effect is not statistically significant. The relationship between other covariates and episode-level 
TABLE 2 Regression Results at the Episode Level with Total Cost per Episode as the Outcome ${ }^{a}$

\begin{tabular}{|c|c|c|c|c|c|c|c|c|c|c|}
\hline & \multicolumn{10}{|c|}{ Cancer Type } \\
\hline & \multicolumn{2}{|l|}{$\mathrm{BC}$} & \multicolumn{2}{|l|}{ NSCLC } & \multicolumn{2}{|l|}{ RCC } & \multicolumn{2}{|l|}{ MM } & \multicolumn{2}{|l|}{ CML } \\
\hline & $\begin{array}{l}\text { Coefficient } \\
\text { (95\% CI) }\end{array}$ & $P$ Value & $\begin{array}{l}\text { Coefficient } \\
\text { (95\% CI) }\end{array}$ & $P$ Value & $\begin{array}{l}\text { Coefficient } \\
\text { (95\% CI) }\end{array}$ & $P$ Value & $\begin{array}{l}\text { Coefficient } \\
\text { (95\% CI) }\end{array}$ & $P$ Value & $\begin{array}{l}\text { Coefficient } \\
(95 \% \mathrm{CI})\end{array}$ & $P$ Value \\
\hline Age & $\begin{array}{c}0.016 \\
(-0.006-0.038) \\
\end{array}$ & 0.153 & $\begin{array}{c}0.036 \\
(0.021-0.051) \\
\end{array}$ & $<0.001$ & $\begin{array}{c}0.028 \\
(-0.026-0.082) \\
\end{array}$ & 0.312 & $\begin{array}{c}0.037 \\
(0.019-0.055) \\
\end{array}$ & $<0.001$ & $\begin{array}{c}0.054 \\
(0.022-0.086)\end{array}$ & $<0.001$ \\
\hline Age-squared & $\begin{array}{c}-0.00021 \\
(-0.00036- \\
-0.00006)\end{array}$ & 0.006 & $\begin{array}{c}-0.00034 \\
(-0.00045- \\
-0.00023)\end{array}$ & $<0.001$ & $\begin{array}{c}-0.00026 \\
(-0.00064- \\
0.00012)\end{array}$ & 0.180 & $\begin{array}{l}-0.00036 \\
(-0.00048- \\
-0.00022)\end{array}$ & $<0.001$ & $\begin{array}{l}-0.00050 \\
(-0.00073- \\
-0.00026)\end{array}$ & $<0.001$ \\
\hline Maleb $^{b}$ & \multicolumn{2}{|l|}{ NA } & $\begin{array}{c}0.032 \\
(0.010-0.054)\end{array}$ & 0.004 & $\begin{array}{c}0.125 \\
(0.022-0.229)\end{array}$ & 0.017 & $\begin{array}{c}0.048 \\
(0.011-0.085) \\
\end{array}$ & 0.012 & $\begin{array}{c}0.029 \\
(-0.064-0.121)\end{array}$ & 0.547 \\
\hline CCI score & $\begin{array}{c}0.123 \\
(0.085-0.161)\end{array}$ & $<0.001$ & $\begin{array}{c}0.031 \\
(0.015-0.047) \\
\end{array}$ & $<0.001$ & $\begin{array}{c}0.041 \\
(-0.021-0.102) \\
\end{array}$ & 0.198 & $\begin{array}{c}0.006 \\
(-0.017-0.030) \\
\end{array}$ & 0.590 & $\begin{array}{c}0.077 \\
(0.021-0.133)\end{array}$ & 0.007 \\
\hline CCI score squared & $\begin{array}{c}-0.0044 \\
(-0.01130- \\
0.00263)\end{array}$ & 0.222 & $\begin{array}{c}-0.00222 \\
(-0.00511- \\
0.00067)\end{array}$ & 0.133 & $\begin{array}{c}0.00392 \\
(-0.00771- \\
0.01560)\end{array}$ & 0.509 & $\begin{array}{c}-0.00013 \\
(-0.00428- \\
0.00402)\end{array}$ & 0.951 & $\begin{array}{c}-0.00559 \\
(-0.01460- \\
0.00346)\end{array}$ & 0.226 \\
\hline $\begin{array}{l}\text { Dual eligible for } \\
\text { Medicaidd }\end{array}$ & $\begin{array}{c}0.067 \\
(0.008-0.127) \\
\end{array}$ & 0.027 & $\begin{array}{c}-0.026 \\
(-0.051-0.000) \\
\end{array}$ & 0.046 & $\begin{array}{c}0.011 \\
(-0.104-0.126) \\
\end{array}$ & 0.852 & $\begin{array}{c}-0.012 \\
(-0.055-0.030)\end{array}$ & 0.574 & $\begin{array}{c}0.055 \\
(-0.059-0.169) \\
\end{array}$ & 0.343 \\
\hline $\begin{array}{l}\text { Disability entitlement } \\
\text { for Medicare }\end{array}$ & $\begin{array}{c}0.018 \\
(-0.067-0.102) \\
\end{array}$ & 0.682 & $\begin{array}{c}-0.021 \\
(-0.054-0.012)\end{array}$ & 0.218 & $\begin{array}{c}-0.001 \\
(-0.155-0.153)\end{array}$ & 0.989 & $\begin{array}{c}-0.040 \\
(-0.097-0.018)\end{array}$ & 0.176 & $\begin{array}{c}-0.053 \\
(-0.189-0.083)\end{array}$ & 0.445 \\
\hline $\begin{array}{l}\text { Died during } \\
\text { episode }^{b}\end{array}$ & $\begin{array}{c}0.317 \\
(0.258-0.376)\end{array}$ & $<0.001$ & $\begin{array}{c}-0.208 \\
(-0.229--0.187) \\
\end{array}$ & $<0.001$ & $\begin{array}{c}-0.026 \\
(-0.110-0.058)\end{array}$ & 0.540 & $\begin{array}{c}-0.163 \\
(-0.214--0.111)\end{array}$ & $<0.001$ & $\begin{array}{c}0.000 \\
(-0.129-0.128) \\
\end{array}$ & 0.998 \\
\hline $\begin{array}{l}\text { Number of } \\
\text { previous episodes }\end{array}$ & $\begin{array}{c}-0.117 \\
(-0.128--0.106)\end{array}$ & $<0.001$ & $\begin{array}{c}-0.022 \\
(-0.032--0.012)\end{array}$ & $<0.001$ & $\begin{array}{c}-0.058 \\
(-0.086--0.030)\end{array}$ & $<0.001$ & $\begin{array}{c}-0.017 \\
(-0.024--0.010)\end{array}$ & $<0.001$ & $\begin{array}{c}-0.002 \\
(-0.014-0.010) \\
\end{array}$ & 0.782 \\
\hline HRRs & & & & Fixed eff & ects of HRRs inclu & aded in al & 1 models $^{\mathrm{e}}$ & & & \\
\hline \multicolumn{11}{|l|}{ Stage of disease ${ }^{c}$} \\
\hline Stage 1 & \multicolumn{2}{|l|}{ NA } & \multicolumn{2}{|c|}{ Reference } & \multicolumn{2}{|l|}{ Reference } & \multicolumn{2}{|l|}{ NA } & \multicolumn{2}{|l|}{ NA } \\
\hline Stage 2 & \multicolumn{2}{|l|}{ NA } & $\begin{array}{c}0.056 \\
(0.000-0.111)\end{array}$ & 0.050 & $\begin{array}{c}0.448 \\
(0.245-0.651)\end{array}$ & $<0.001$ & \multicolumn{2}{|l|}{ NA } & \multicolumn{2}{|l|}{ NA } \\
\hline Stage 3 & \multicolumn{2}{|l|}{ Reference } & $\begin{array}{c}0.209 \\
(0.171-0.247) \\
\end{array}$ & $<0.001$ & $\begin{array}{c}0.394 \\
(0.232-0.557) \\
\end{array}$ & $<0.001$ & \multicolumn{2}{|l|}{ NA } & \multicolumn{2}{|l|}{ NA } \\
\hline Stage 4 & $\begin{array}{c}0.420 \\
(0.362-0.478)\end{array}$ & $<0.001$ & $\begin{array}{c}0.271 \\
(0.233-0.308)\end{array}$ & $<0.001$ & $\begin{array}{c}0.679 \\
(0.567-0.791)\end{array}$ & $<0.001$ & \multicolumn{2}{|l|}{ NA } & \multicolumn{2}{|l|}{ NA } \\
\hline
\end{tabular}

${ }^{a}$ Coefficients were acquired from generalized linear models with a log link and gamma distribution with total cost per episode (adjusted for Medicare payment rate differentials and rate changes over time) as the outcome. Indicator variables were included for each HRR.

bReference group for analysis was "female."

"For BC, only stages 3 and 4 were included in the study. For MM and CML, standard American Joint Committee on Cancer staging does not apply and was not acquired from the data. For stage variables, "reference" indicates that a given stage was the reference group for analysis.

"Reference group for analysis is "no."

eWald test $P<0.001$ for all cancer types, rejecting the null hypothesis that all HRR coefficients $=0$ and indicating significance in improving model fit.

$B C=$ advanced breast cancer (stages 3 and 4); CCI =Charlson Comorbidity Index; CI=confidence interval; $C M L=$ chronic myeloid leukemia; HRR=hospital referral region; $\mathrm{MM}=$ multiple myeloma; $N A=$ not available; NSCLC =non-small cell lung cancer; $R C C=$ renal cell carcinoma .

spending is more varied. For example, dual eligibility for Medicaid was associated with $6.7 \%$ (coefficient $=0.067$, $P=0.027$ ) higher spending in advanced $B C$ but $2.6 \%$ lower spending in NSCLC (coefficient $=-0.026, P=0.046$ ).

Our analysis showed that, after controlling for patient characteristics, there were significant differences in standardized spending per episode across HRRs for each of the 5 cancers (Figure 1). A Wald test determined that including regional effects created a statistically significant improvement in the model fit for each cancer and indicated that the region coefficients were significantly different from one another and simultaneously not equal to zero (Wald test $P<0.001$ for all cancers). Compared with spending at the 20th percentile episode, spending at the 80th percentile ranged from 25\% higher ( $\$ 57,392$ vs. $\$ 45,995$ for MM) to $47 \%$ higher $(\$ 36,920$ vs. $\$ 25,127$ for RCC), indicating that the amount of variation in spending was the least for MM and the greatest for RCC. The interquartile ranges varied from approximately $\$ 5,400$ for advanced BC to about $\$ 11,000$ for CML

Next, we examined the relationship between the patient-level hazard of death - that is, the probability of death at any time conditional on survival up to that time-and regional per episode standardized spending and other covariates that described the patient. The estimated coefficients appear in Table 3. Our 


\section{FIGURE 1 Regional Variation in Standardized Spending for 5 Types of Cancer (2013 USD)}

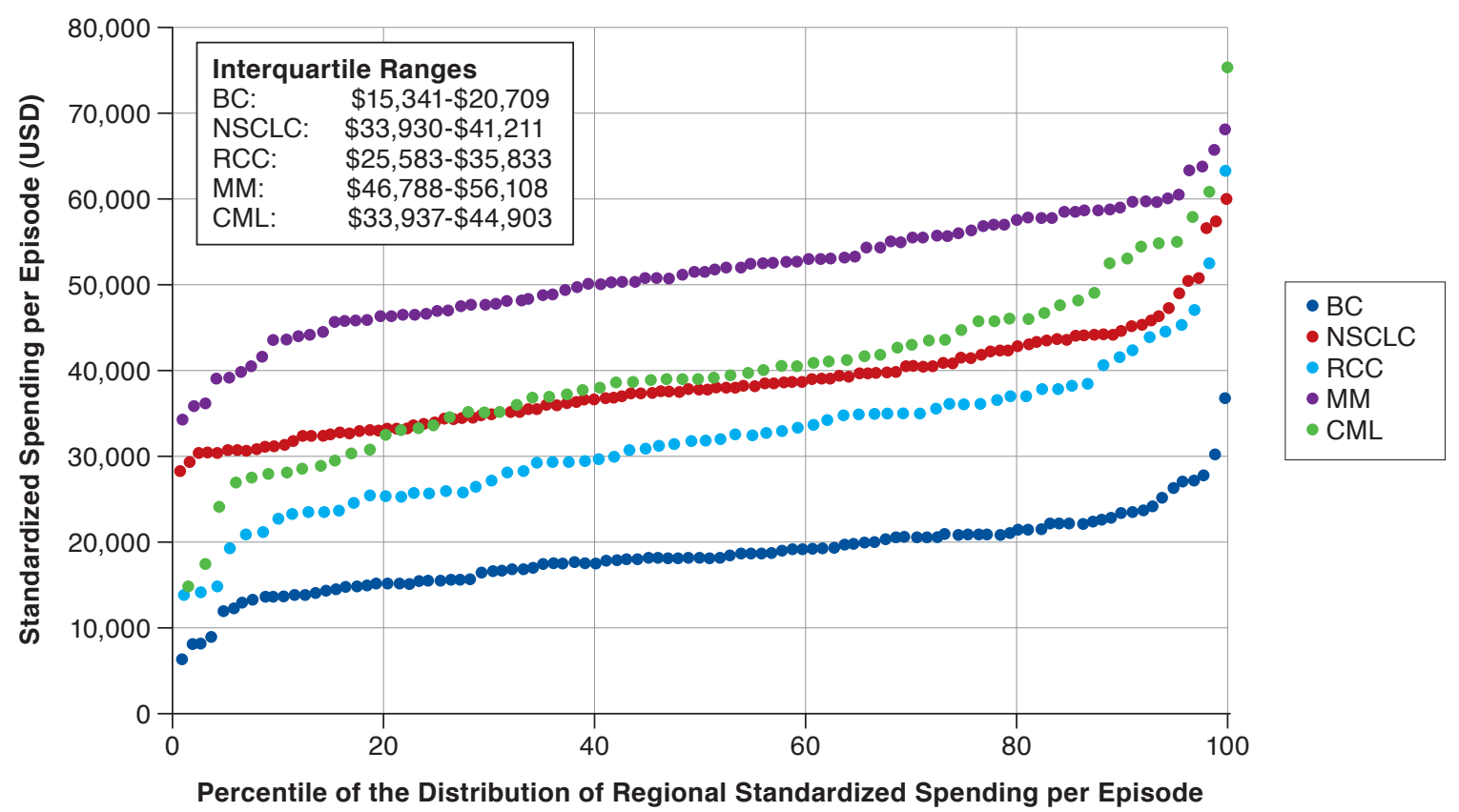

$B C=$ advanced breast cancer (stages 3 and 4); CML = chronic myeloid leukemia; $M M=$ multiple myeloma; NSCLC = non-small cell lung cancer; RCC = renal cell carcinoma; USD $=2013$ U.S. dollars.

specifications passed the Schoenfeld residuals test for proportional hazards. The effects of covariates generally accorded with expectations. As one might expect, risk of death increased with more advanced stages of cancer at time of diagnosis, but that increased risk declined slightly over time. Interestingly, dual eligibility for Medicaid was also associated with a statistically significant increased probability of death, ranging from a 14\% increase for NSCLC patients (hazard ratio $(\mathrm{HR})=1.14, P<0.001$ ) to a $34 \%$ increase for $\mathrm{MM}$ patients $(\mathrm{HR}=1.34, P=0.001)$ in all cancer types except CML (HR=0.82, $P=0.317)$.

Figure 2 illustrates the estimated effect of spending per episode on the hazard of death. We found a statistically significant reduction in the hazard of death for NSCLC and MM when there was greater spending per episode. Specifically, the hazard rate of dying for patients with NSCLC and MM, respectively, decreased by $7 \%(\mathrm{HR}=0.93, \mathrm{P}=0.006)$ and $13 \%$ ( $\mathrm{HR}=0.87, P=0.019$ ) for a $\$ 10,000$ increase in standardized episode spending. For the 3 remaining cancer sites, results suggested a reduction in hazard of death in RCC and CML and an increase in advanced BC as standardized spending increased, but none of these results was statistically significant at the 5\% level. After multiple comparisons adjustment using the Benjamini-Hochberg procedure with an FDR of $5 \%$, the effects of increased spending on improved survival for NSCLC and MM remained statistically significant.

\section{Discussion}

$\overline{\text { Our results on standardized spending per episode across HRRs }}$ bear a similarity to other analyses of geographic variations in health care by demonstrating that such variations exist and are significant. When coupled with findings of no systematic variation in outcomes, previous analyses have been interpreted as evidence of waste in the system. Brooks et al. (2013) examined the extent of regional variation in cancer spending in advanced cancer and found substantial regional variation in Medicare spending categories for advanced non-small cell lung, colorectal, pancreatic, breast, and prostate cancers. ${ }^{11}$ The authors also examined the relationship between spending and cohort-level survival outcomes, finding no consistent or statistically significant relationship. In contrast, Keating et al. (2012), as with our study, found greater survival among lung cancer patients in higher-spending regions. ${ }^{12}$ Hassett et al. (2014) focused on breast cancer and, similar to Brooks et al. and our study, found no correlation for that cancer type between survival and either spending level or quality of care. ${ }^{13}$

Our study differs in several ways from earlier studies of geographic variation in cancer care. ${ }^{11-17}$ Unlike previous work, we used the OCM definition of an episode. Thus, our analysis can more readily speak to the potential effects of reducing spending during chemotherapy-centered episodes such as those used by 


\begin{tabular}{|c|c|c|c|c|c|c|c|c|c|c|}
\hline & \multicolumn{10}{|c|}{ Cancer Type } \\
\hline & \multicolumn{2}{|l|}{$\mathrm{BC}$} & \multicolumn{2}{|c|}{ NSCLC } & \multicolumn{2}{|l|}{ RCC } & \multicolumn{2}{|l|}{ MM } & \multicolumn{2}{|l|}{ CML } \\
\hline & $\begin{array}{c}\text { HR } \\
(95 \% \mathrm{CI})\end{array}$ & $P$ Value & $\begin{array}{c}\text { HR } \\
(95 \% \mathrm{CI})\end{array}$ & $P$ Value & $\begin{array}{c}\text { HR } \\
(95 \% \mathrm{CI})\end{array}$ & $P$ Value & $\begin{array}{c}\text { HR } \\
(95 \% \mathrm{CI})\end{array}$ & $P$ Value & $\begin{array}{c}\text { HR } \\
(95 \% \mathrm{CI})\end{array}$ & $P$ Value \\
\hline $\begin{array}{l}\text { Standardized spending for a } \\
\text { region (in } \$ 10,000 \text { s USD) }\end{array}$ & $\begin{array}{c}1.06 \\
(0.91-1.24)\end{array}$ & 0.442 & $\begin{array}{c}0.93 \\
(0.88-0.98) \\
\end{array}$ & 0.006 & $\begin{array}{c}0.94 \\
(0.81-1.10)\end{array}$ & 0.455 & $\begin{array}{c}0.87 \\
(0.77-0.98)\end{array}$ & 0.019 & $\begin{array}{c}0.87 \\
(0.69-1.09) \\
\end{array}$ & 0.216 \\
\hline Age at diagnosis & $\begin{array}{c}0.91 \\
(0.87-0.96) \\
\end{array}$ & $<0.001$ & $\begin{array}{c}0.94 \\
(0.91-0.97)\end{array}$ & $<0.001$ & $\begin{array}{c}0.86 \\
(0.77-0.96)\end{array}$ & 0.009 & $\begin{array}{c}0.95 \\
(0.87-1.04)\end{array}$ & 0.272 & $\begin{array}{c}1.01 \\
(0.86-1.19)\end{array}$ & 0.882 \\
\hline Age at diagnosis squared & $\begin{array}{c}1.0009 \\
(1.0005- \\
1.0012)\end{array}$ & $<0.001$ & $\begin{array}{c}1.0006 \\
(1.0003- \\
1.0008)\end{array}$ & $<0.001$ & $\begin{array}{c}1.0012 \\
(1.0004- \\
1.0020)\end{array}$ & 0.002 & $\begin{array}{c}1.0007 \\
(1.0001- \\
1.0013)\end{array}$ & 0.031 & $\begin{array}{c}1.0004 \\
(0.9993- \\
1.0014)\end{array}$ & 0.519 \\
\hline Male $^{c}$ & Omitte & & $\begin{array}{c}1.32 \\
(1.26-1.38)\end{array}$ & $<0.001$ & $\begin{array}{c}1.20 \\
(0.99-1.45)\end{array}$ & 0.062 & $\begin{array}{c}1.08 \\
(0.94-1.24)\end{array}$ & 0.258 & $\begin{array}{c}1.21 \\
(0.86-1.71)\end{array}$ & 0.277 \\
\hline Medicaid eligibled & $\begin{array}{c}1.26 \\
(1.10-1.44)\end{array}$ & 0.001 & $\begin{array}{c}1.14 \\
(1.08-1.20)\end{array}$ & $<0.001$ & $\begin{array}{c}1.28 \\
(1.02-1.59)\end{array}$ & 0.030 & $\begin{array}{c}1.34 \\
(1.13-1.58)\end{array}$ & 0.001 & $\begin{array}{c}0.82 \\
(0.57-1.20)\end{array}$ & 0.317 \\
\hline $\begin{array}{l}\text { Medicare entitlement through } \\
\text { disability }\end{array}$ & $\begin{array}{c}1.41 \\
(1.19-1.67)\end{array}$ & $<0.001$ & $\begin{array}{c}1.11 \\
(1.04-1.19)\end{array}$ & 0.001 & $\begin{array}{c}0.92 \\
(0.67-1.28)\end{array}$ & 0.632 & $\begin{array}{c}1.15 \\
(0.92-1.45)\end{array}$ & 0.227 & $\begin{array}{c}1.19 \\
(0.72-1.97)\end{array}$ & 0.490 \\
\hline CCI score & $\begin{array}{c}1.21 \\
(1.11-1.32)\end{array}$ & $<0.001$ & $\begin{array}{c}1.07 \\
(1.03-1.11) \\
\end{array}$ & $<0.001$ & $\begin{array}{c}1.07 \\
(0.94-1.22)\end{array}$ & 0.305 & $\begin{array}{c}1.10 \\
(0.99-1.21)\end{array}$ & 0.076 & $\begin{array}{c}1.10 \\
(0.88-1.37)\end{array}$ & 0.415 \\
\hline CCI score squared & $\begin{array}{c}0.9790 \\
(0.9640- \\
0.9950) \\
\end{array}$ & 0.010 & $\begin{array}{c}0.9957 \\
(0.9891- \\
1.0024) \\
\end{array}$ & 0.212 & $\begin{array}{c}0.9948 \\
(0.9726- \\
1.0176) \\
\end{array}$ & 0.655 & $\begin{array}{c}0.9977 \\
(0.9800- \\
1.0156) \\
\end{array}$ & 0.797 & $\begin{array}{c}1.0010 \\
(0.9710- \\
1.0318) \\
\end{array}$ & 0.951 \\
\hline \multicolumn{11}{|l|}{ Stage at diagnosis } \\
\hline Stage 1 (fixed) & \multicolumn{2}{|l|}{ NA } & \multicolumn{2}{|c|}{ Reference } & \multicolumn{2}{|c|}{ Reference } & \multicolumn{2}{|l|}{ NA } & \multicolumn{2}{|l|}{ NA } \\
\hline Stage 1 (time varying) & \multicolumn{2}{|l|}{ NA } & \multicolumn{2}{|c|}{ Reference } & \multicolumn{2}{|c|}{ Reference } & \multicolumn{2}{|l|}{ NA } & \multicolumn{2}{|l|}{ NA } \\
\hline Stage 2 (fixed) & \multicolumn{2}{|l|}{ NA } & $\begin{array}{c}1.31 \\
(1.09-1.57)\end{array}$ & 0.004 & $\begin{array}{c}2.15 \\
(1.08-4.31)\end{array}$ & 0.028 & \multicolumn{2}{|l|}{ NA } & \multicolumn{2}{|l|}{ NA } \\
\hline Stage 2 (time varying) & \multicolumn{2}{|l|}{ NA } & $\begin{array}{c}1.00 \\
(0.83-1.20)\end{array}$ & 0.960 & $\begin{array}{c}1.01 \\
(0.05-2.02)\end{array}$ & 0.983 & \multicolumn{2}{|l|}{ NA } & \multicolumn{2}{|l|}{ NA } \\
\hline Stage 3 (fixed) & \multicolumn{2}{|c|}{ Reference } & $\begin{array}{c}3.06 \\
(2.71-3.45)\end{array}$ & $<0.001$ & $\begin{array}{c}2.47 \\
(1.48-4.12)\end{array}$ & 0.001 & \multicolumn{2}{|l|}{ NA } & \multicolumn{2}{|l|}{ NA } \\
\hline Stage 3 (time varying) & \multicolumn{2}{|c|}{ Reference } & $\begin{array}{c}0.98 \\
(0.87-1.10) \\
\end{array}$ & 0.739 & $\begin{array}{c}1.00 \\
(0.60-1.67) \\
\end{array}$ & 0.990 & \multicolumn{2}{|l|}{ NA } & \multicolumn{2}{|l|}{ NA } \\
\hline Stage 4 (fixed) & $\begin{array}{c}3.76 \\
(3.14-4.51) \\
\end{array}$ & $<0.001$ & $\begin{array}{c}6.72 \\
(5.97-7.58) \\
\end{array}$ & $<0.001$ & $\begin{array}{c}12.50 \\
(8.09-19.33) \\
\end{array}$ & $<0.001$ & NA & & NA & \\
\hline Stage 4 (time varying) & $\begin{array}{c}0.99 \\
(0.82-1.19)\end{array}$ & 0.906 & $\begin{array}{c}0.97 \\
(0.86-1.09) \\
\end{array}$ & 0.561 & $\begin{array}{c}0.97 \\
(0.63-1.50)\end{array}$ & 0.887 & NA & & NA & \\
\hline Race/ethnicity & & & & & & & & & & \\
\hline White & Referen & & Referen & & Referen & & Referen & & Referenc & \\
\hline Black & $\begin{array}{c}1.10 \\
(0.92-1.30) \\
\end{array}$ & 0.301 & $\begin{array}{c}1.00 \\
(0.92-1.07) \\
\end{array}$ & 0.930 & $\begin{array}{c}1.17 \\
(0.82-1.67) \\
\end{array}$ & 0.380 & $\begin{array}{c}0.99 \\
(0.80-1.22)\end{array}$ & 0.890 & $\begin{array}{c}0.97 \\
(0.57-1.64)\end{array}$ & 0.914 \\
\hline Hispanic & $\begin{array}{c}0.83 \\
(0.59-1.17) \\
\end{array}$ & 0.294 & $\begin{array}{c}1.00 \\
(0.85-1.18) \\
\end{array}$ & 0.964 & $\begin{array}{c}0.86 \\
(0.53-1.40) \\
\end{array}$ & 0.540 & $\begin{array}{c}0.93 \\
(0.64-1.36) \\
\end{array}$ & 0.714 & $\begin{array}{c}2.64 \\
(0.07-100.09) \\
\end{array}$ & 0.601 \\
\hline Asian & $\begin{array}{c}0.93 \\
(0.67-1.30) \\
\end{array}$ & 0.670 & $\begin{array}{c}0.90 \\
(0.82-1.00)\end{array}$ & 0.040 & $\begin{array}{c}1.03 \\
(0.69-1.54)\end{array}$ & 0.873 & $\begin{array}{c}0.98 \\
(0.72-1.34)\end{array}$ & 0.922 & $\begin{array}{c}0.88 \\
(0.42-1.83)\end{array}$ & 0.728 \\
\hline Native American & NC & & $\begin{array}{c}1.10 \\
(0.68-1.78)\end{array}$ & 0.710 & $\begin{array}{c}2.46 \\
(1.23-4.94)\end{array}$ & 0.011 & NC & & $\mathrm{NC}$ & \\
\hline Other & $\begin{array}{c}1.03 \\
(0.64-1.67)\end{array}$ & 0.888 & $\begin{array}{c}0.90 \\
(0.77-1.04)\end{array}$ & 0.151 & $\begin{array}{c}1.15 \\
(0.65-2.04) \\
\end{array}$ & 0.634 & $\begin{array}{c}0.96 \\
(0.55-1.67)\end{array}$ & 0.884 & $\mathrm{NC}$ & \\
\hline Unknown & NC & & $\begin{array}{c}0.97 \\
(0.56-1.69) \\
\end{array}$ & 0.926 & NC & & NC & & $\mathrm{NC}$ & \\
\hline $\begin{array}{l}{ }^{a} \text { Confidence intervals and } P \text { valu } \\
\text { bSome values were not calculable } \\
{ }^{c} \text { Reference group for analysis is " } \\
{ }^{d} \text { Reference group for analysis is " } \\
B C=\text { advanced breast cancer (sta } \\
\text { available; NC = not calculable; N. }\end{array}$ & $\begin{array}{l}\text { bootstrapp } \\
\text { isufficient s }\end{array}$ & $\begin{array}{l}\text { standar } \\
\text { uple size }\end{array}$ & $\begin{array}{l}\text { al; } C M L=c \\
R C C=\text { reno }\end{array}$ & by "NC". & $C_{\Gamma}$ & 1 & 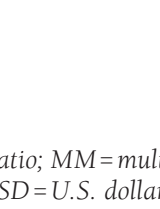 & le & $A=n o t$ & \\
\hline
\end{tabular}




\section{FIGURE 2 Hazard Ratios for a $\$ 10,000$ Increase in} HRR Standardized Spending

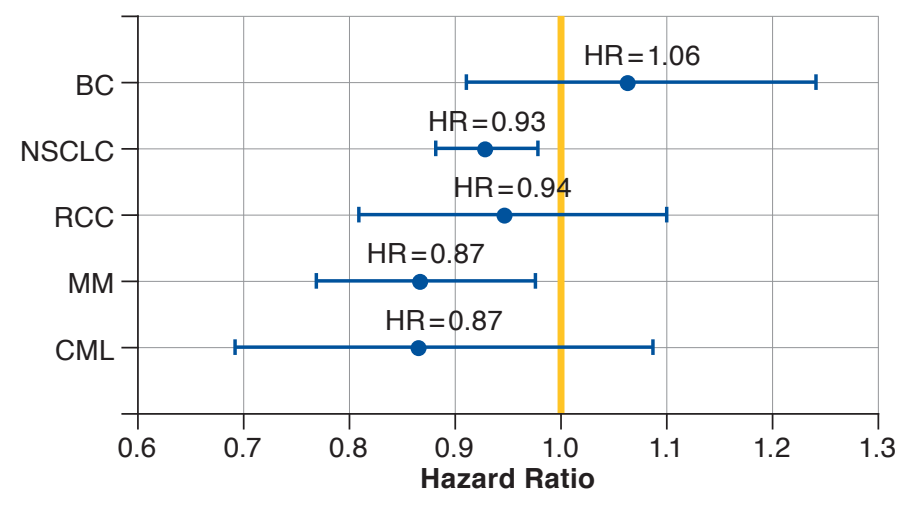

Notes: The figure depicts the ratio of the risk of death between 2 individuals whose values of HRR standardized spending differ by 1 unit (\$10,000 in 2013 USD) holding constant the values of the other covariates, with the numerator representing the risk at the higher value of spending. Whisker plots represent 95\% CIs; hence, where the CI does not overlap the value 1, results are statistically significant $(P<0.05)$. Results for NSCLC and MM remain statistically significant after applying the Benjamini-Hochberg procedure on the P values with a false discovery rate of $5 \%$. $B C=$ advanced breast cancer; $C I=$ confidence interval; $C M L=$ chronic myeloid leukemia; $H R=$ hazard ratio; $H R R=$ hospital referral region; $M M=$ multiple myeloma; NSCLC = non-small cell lung cancer; RCC = renal cell carcinoma; USD =U.S. dollars.

the OCM. We also differ from other studies in some of the cancer types that we included-to our knowledge, we are the first to consider RCC, MM, and CML. We also used more recent data and included Medicare Part D chemotherapy costs (and Part D drug costs more generally).

For 2 of the cancer types we studied (NSCLC and MM), we found a beneficial and statistically significant effect of spending on survival, with greater standardized spending per episode at the HRR level associated with lower hazard of death. While these results do not rule out the existence of inefficiencies, they do indicate that greater spending is associated with improved survival outcomes. Unless further studies are able to separate out effective from ineffective spending, the implication is that across-the-board reductions in spending may reduce survival, on average, for some cancer types.

One might ask whether the additional survival is worth the cost. Given the estimated HRs, one can approximate the additional life expectancy, compared with the median, from a $\$ 10,000$ increase in standardized spending per episode. For NSCLC, an additional $\$ 10,000$ in standardized episode spending was associated with a 1.53 month increase in median survival. After adjusting for number of episodes per patient, that translates to about $\$ 107,000$ per additional life-year. Utility adjustments for reduced quality of life for patients with this cancer vary across the literature, but depending on the utility value chosen, the range of cost per quality-adjusted life-year
(QALY) is about $\$ 160,000-\$ 228,000 \cdot{ }^{18-20}$ For MM, an additional $\$ 10,000$ in spending is associated with an increase in median survival estimated at 11 months, or after adjusting for multiple episodes per patient, a cost of $\$ 36,000$ per additional life-year. Applying a utility adjustment for a myeloma patient yields a cost per added QALY number of $\$ 69,000 .{ }^{21}$ Details on these calculations are in Appendix B (available in online article). Neumann et al. (2014) recommended that analysts use a range of thresholds between $\$ 50,000$ and $\$ 200,000$ per QALY for defining cost-effective care. ${ }^{22}$ Hence, the additional spending is near the higher end of the suggested cost-effectiveness range of cost per QALY thresholds for NSCLC and near the lower, more favorable, end for MM.

With regard to the OCM specifically, 2 important points should be recognized. First, a key policy lever in the OCM is the setting of the spending thresholds, below which a practice receives a performance-based payment $(2.75 \%$ or $4 \%$ below the otherwise calculated target for a practice depending on whether two-sided or one-sided risk is used). Such thresholds are incentives to reduce spending, and our results suggest that, in setting these thresholds for specific types of cancer, policymakers should consider the potential for blunt spending reductions to affect survival. Second, in setting its targets for practices, the OCM does allow differences based on the HRR of the practice. Thus, the OCM allows the current amount of geographic variation to continue but encourages reductions in spending from those baseline differentials. Our analysis simply used the existing geographic variation as a tool for measuring the effect of episode spending on survival.

Our results are relevant to the general set of policies that aim to provide cancer care at lower spending levels than otherwise. Although we defined episodes in the same way as the OCM, we defined spending slightly differently. Our study included spending on patient copays and deductibles and all spending on Part D drugs, although the OCM model excludes some of these sources. We took this approach so that our results would have more general applicability and could answer the broader questions of whether and how systemwide spending is associated with survival. This also has the effect of providing a broader, societal perspective on total spending, as is currently recommended for economic evaluations in health care. ${ }^{23}$

While we did find evidence of an association between improved survival outcomes and higher episode spending in some cases, our analysis did not identify the specific differences in treatment choices that explain the survival differences. As in the OCM, our measure of episode spending included all types of services, although chemotherapy costs were likely a substantial fraction of costs in these episodes. Several expensive therapy options existed in the time frame of our analysis for all the cancer types we studied (in the case of NSCLC, these included crizotinib, erlotinib, and gefitinib, and for MM, these included bortezomib, lenalidomide, and 
carfilzomib). Future research using real-world evidence may be able to distinguish differences in practice patterns in chemotherapy choice and document survival differences.

Use of quality measures may be one strategy for encouraging more efficient spending in APMs and related strategies (e.g., clinical pathways) that also aim to limit spending. The OCM payment methodology bases the amount of a performancebased payment on a practice's performance on several quality measures. It is not yet known how the use of the particular quality measures in the OCM payment formula affects outcomes. Our analysis suggests that physicians, pharmacists, and other health professionals involved in the OCM and in other APMs should carefully consider the areas in which they reduce spending and the potential effect on outcomes, especially in NSCLC and MM.

\section{Limitations}

As with all analyses, our study has limitations. First, our data were limited to regions available in the SEER registries, which cover only about $28 \%$ of the U.S. population, and to the Medicare population. Hence, the analysis may not be representative of the nation as a whole or to APMs outside of Medicare; however, because the OCM is a Medicare initiative for the traditional fee-for-service population, a Medicare sample such as ours is appropriate to the OCM.

In addition, the SEER-Medicare database, which is maintained by the National Cancer Institute, provided us with wellregarded measures of diagnosis, staging, and diagnosis dates and also covered a wide variety of geographic regions. Because we studied patients enrolled in Medicare Parts A, B, and D, our results may not be representative of the entire Medicareeligible population, if those who opt into Part D are systematically different from others.

Also, while we structured episodes of cancer care to reflect the definition used in the OCM, our data were drawn from a period ending in 2013. Therefore, any recent changes in treatment patterns for the included cancers are not reflected here.

Finally, although survival is clearly an important outcome for assessing cancer treatment, another limitation is that we restricted our study to only that measure. It is possible that other endpoints or patient-reported outcomes, such as quality of life and satisfaction, are also affected by spending differences.

\section{Conclusions}

$\overline{\text { After controlling for differences caused by Medicare payment }}$ differentials and patient characteristics, we found statistically significant differences in spending per episode across geographic regions in 5 different types of cancer. While such differences suggest the possibility that inefficient or wasteful spending is occurring, we also found evidence that greater spending is associated with improved survival in NSCLC and $\mathrm{MM}$, with a similar but statistically insignificant association in
2 other cancer types. The OCM and other future payment models that move away from fee-for-service represent a move toward a new variant of Medicare managed care in which practices are faced with a new set of incentives within "traditional" Medicare. Physicians, pharmacists, other health care professionals, and policymakers should maintain awareness of how curbing resource use in particular areas may adversely affect outcomes and build incentive and management mechanisms accordingly.

\section{Authors}

JAMES BAUMGARDNER, PhD; AHVA SHAHABI, PhD; MARK LINTHICUM, MPP; and SEANNA VINE, MPH, Precision Health Economics, Los Angeles, California. CHRISTOPHER ZACKER, RPh, PhD, Novartis Pharmaceuticals, East Hanover, New Jersey, and DARIUS LAKDAWALLA, PhD, Leonard D. Schaeffer Center for Health Policy E Economics, University of Southern California, Los Angeles.

AUTHOR CORRESPONDENCE: James Baumgardner, PhD, Precision Health Economics, 11100 Santa Monica Blvd., Ste. 500, Los Angeles, CA 90025. Tel.: 310.984.7781;

E-mail: james.baumgardner@PrecisionHealthEconomics.com.

\section{DISCLOSURES}

Funding for this research was provided by Novartis Pharmaceuticals to Precision Health Economics in support of research design, analysis, and technical writing services. The funder provided input on study design and comments on the draft report. Baumgardner, Shahabi, and Linthicum are employees of Precision Health Economics (PHE), a health care consultancy to the insurance and life science industries, including firms that market oncology therapies. Vine was an employee of PHE at the time of this research. Zacker is an employee of and shareholder in Novartis Pharmaceuticals. Lakdawalla is a consultant to PHE and holds equity in its parent company, Precision Medicine Group.

\section{ACKNOWLEDGMENTS}

This study used the linked SEER-Medicare database. The interpretation and reporting of these data are the sole responsibility of the authors. The authors acknowledge the efforts of the National Cancer Institute; the Office of Research, Development and Information, CMS; Information Management Services; and the Surveillance, Epidemiology, and End Results (SEER) Program tumor registries in the creation of the SEER-Medicare database. The authors also thank Amitabh Chandra, Sarah Beers, Michelle Brauer, Sarah Green, Caroline Huber, Aubrey Love, Joanna MacEwan, and Lara Yoon for their advice and assistance.

\section{REFERENCES}

1. Newcomer LN, Gould B, Page RD, Donelan SA, Perkins M. Changing physician incentives for affordable, quality cancer care: results of an episode payment model. J Oncol Pract. 2014;10(5):322-26.

2. Kline RM, Bazell C, Smith E, Schumacher H, Rajkumar R, Conway PH. Centers for Medicare and Medicaid Services: using an episode-based payment model to improve oncology care. J Oncol Pract. 2015;11(2):114-16. 
3. Center for Medicare \& Medicaid Innovation. OCM performance-based payment methodology (version 2.1). Prepared by RTI International and Actuarial Research Corporation. In: Oncology care model performance periods 1 and 2 payment methodology [zip file]. December 27, 2017. Available at: https://innovation.cms. gov/initiatives/oncology-care/. May 8, 2018.

4. Clough JD, Kamal AH. Oncology care model: short-and long-term considerations in the context of broader payment reform. J Oncol Pract. 2015;11(4):319-21.

5. Skinner J. Causes and consequences of regional variations in health care. In: Pauly MV, McGuire TG, Barros PP, eds. Handbook of Health Economics, Vol 2. Waltham, MA: North Holland; 2011:45-93.

6. Congressional Budget Office. Geographic variation in health care spending. February 2008. Available at: https://www.cbo.gov/sites/default/ files/110th-congress-2007-2008/reports/02-15-geoghealth_0.pdf. Accessed April 27, 2018.

7. IOM Committee on Geographic Variation in Health Care Spending and Promotion of High-value Care. Geographic variation in spending, utilization and quality: Medicare and Medicaid beneficiaries. Prepared by Acumen. May 2013. Available at: http://www.nationalacademies.org/hmd/ /media/ Files/Report\%20Files/2013/Geographic-Variation/Sub-Contractor/AcumenMedicare-Medicaid.pdf. Accessed April 27, 2018.

8. The Dartmouth Atlas of Health Care. Data by region. About our regions: hospital referral regions. Retrieved June 26, 2017. Available at: http://www. dartmouthatlas.org/data/region/. Accessed April 27, 2018.

9. Centers for Medicare \& Medicaid Services. Medicare data for the geographic variation public use file: a methodological overview. March 2017. Available at: https://www.cms.gov/Research-Statistics-Data-and-Systems/ Statistics-Trends-and-Reports/Medicare-Geographic-Variation/Downloads/ Geo_Var_PUF_Methods_Paper.pdf. Accessed April 27, 2018.

10. Benjamini Y, Hochberg Y. Controlling the false discovery rate: a practical and powerful approach to multiple testing. J R Stat Soc Series B Stat Methodol. 1995;57(1):289-300.

11. Brooks GA, Li L, Sharma DB, et al. Regional variation in spending and survival for older adults with advanced cancer. J Natl Cancer Inst. 2013;105(9):634-42.

12. Keating NL, Landrum MB, Lamont EB, Bozeman SR, McNeil BJ. Arealevel variations in cancer care and outcomes. Med Care. 2012;50(5):366-73.

13. Hassett MJ, Neville BA, Weeks JC. The relationship between quality, spending and outcomes among women with breast cancer. J Natl Cancer Inst. 2014;106(10). pii: dju242. Available at: https://academic.oup.com/jnci/ article/106/10/dju242/928111. Accessed May 2, 2018.
14. Brooks GA, Li L, Uno H, Hassett MJ, Landon BE, Schrag D. Acute hospital care is the chief driver of regional spending variation in Medicare patients with advanced cancer. Health Aff (Millwood). 2014;33(10):1793-800.

15. Landrum MB, Meara ER, Chandra A, Guadagnoli E, Keating NL. Is spending more always wasteful? The appropriateness of care and outcomes among colorectal cancer patients. Health Aff (Millwood). 2008;27(1):159-68.

16. Skolarus TA, Ye Z, Zhang S, Hollenbeck BK. Regional differences in early stage bladder cancer care and outcomes. Urology. 2010;76(2):391-96.

17. Fisher ES, Wennberg DE, Stukel TA, Gottlieb DJ, Lucas FL, Pinder EL. The implications of regional variations in Medicare spending. Part 2: health outcomes and satisfaction with care. Ann Intern Med. 2003;138(4):288-98.

18. Doyle S, Lloyd A, Walker M. Health state utility scores in advanced nonsmall cell lung cancer. Lung Cancer. 2008;62(3):374-80.

19. Nafees B, Stafford M, Gavriel S, Bhalla S, Watkins J. Health state utilities for non small cell lung cancer. Health Qual Life Outcomes. 2008;6(1):84.

20. Trippoli S, Vaiani M, Lucioni C, Messori A. Quality of life and utility in patients with non-small cell lung cancer. Pharmacoeconomics. 2001;19(8):855-63.

21. Kharroubi SA, Edlin R, Meads D, Browne C, Brown J, McCabe C. Use of Bayesian Markov Chain Monte Carlo methods to estimate EQ-5D utility scores from EORTC QLQ data in myeloma for use in cost-effectiveness analysis. Med Decis Making. 2015;35(3):351-60.

22. Neumann PJ, Cohen JT, Weinstein MC. Updating cost-effectivenessthe curious resilience of the \$50,000-per-QALY threshold. N Engl J Med. 2014;371(9):796-97.

23. Neumann PJ, Sanders GD, Russell LB, Siegel JE, Ganiats TG, eds. Cost-Effectiveness in Health and Medicine. 2d ed. Oxford: Oxford University Press; 2016.

24. Schousboe JT, Paudel ML, Taylor BC, et al. Estimation of standardized hospital costs from Medicare claims that reflect resource requirements for care: impact for cohort studies linked to Medicare claims. Health Serv Res. 2014:49(3):929-49.

25. Centers for Medicare \& Medicaid Services. Medicare geographic variation. Public use file. 2017. Retrieved May 5, 2017. Available at: https://www. cms.gov/research-statistics-data-and-systems/statistics-trends-and-reports/ medicare-geographic-variation/gv_puf.html. Accessed April 27, 2018.

26. Hanh J. Medicare physician payment updates and the sustainable growth rate (SGR) system. Congressional Research Service R40907. June 12, 2014. Available at: https://greenbook-waysandmeans.house.gov/sites/greenbook.waysandmeans.house.gov/files/R40907_gb.pdf. Accessed May 8, 2018. 


\section{APPENDIX A Further Details: Histology Codes and Standardization for Differences in Medicare Prices}

Study Population: Histology Codes for RCC and NSCLC

In addition to the ICD-O-3 codes reported in the text, the RCC and NSCLC populations were further refined using histology codes (RCC: 8260, 8310, 8312, $8316,8317,8318,8319,8320,8510,8959$. NSCLC: 8012, 8013, 8014, 8022, 8031, 8032, 8033, 8046, 8052, 8070, 8071, 8072, 8073, 8082, 8083, 8084, $8123,8140,8250,8251,8252,8253,8254,8255,8260,8310,8333,8430,8470,8480,8481,8490,8550,8560,8972,8980)$.

\section{Standardization for Differences in Medicare Payment Rates}

Because we fundamentally care about differences in utilization, we adjusted spending for the different prices that Medicare pays for the same services in different regions. For each claim, we adjusted the spending value to a standardized amount so that the same service will be priced at the same dollar value regardless of region or price markups received by particular providers, such as the indirect medical education adjustments or disproportionate share adjustment received by certain hospitals for inpatient care, or adjustments to payment rates to reflect varying wage rates or input costs across different regions.

The standardization method varied based on claim type. For inpatient hospital costs, we proceeded as follows, using LOS and DRG.

\section{Spending Calculation for Inpatient Hospitalizations}

Costs for extra short inpatient hospital stays accompanied by a transfer to another hospital, SNF, or IRF were calculated as:

$\left\{2 \times(\text { Daily Base Amount })_{\text {Unadjusted }}+(\right.$ LOS-1 $\left.) \times(\text { Daily Base Amount })_{\text {Unadjusted }}\right\} \times D R G_{\text {Weight }}+($ Outlier Payment $)$

where weights and geometric mean LOS values were available from CMS by claim year and DRG. The cost of all other inpatient hospitalizations was calculated as:

(Standard Base Amount $)_{\text {Unadjusted }} \times \mathrm{DRG}_{\text {Weight }}+($ Outlier Payment $)$

(These methods for obtaining a standardized spending amount for inpatient claims follow Schousboe et al., 2014.)24

Part B drug claims did not need to be adjusted because of uniform payment nationwide. All other Part A and Part B claims were assigned to a cost-adjustment category to match up with tables developed by CMS to adjust for payment differences at the county level (categories are long-term care hospital, SNF, HHA, hospice, federally qualified health center/rural health center, hospital outpatient, ambulatory surgery center, E + M, procedures, imaging, DME, tests, general outpatient, and ambulance). ${ }^{25}$ The base costs were multiplied by the CMS ratio of standardized to actual cost for the appropriate cost category, FIPS county, and claim year. If the value of the actual or standardized amount was zero or missing for the county, we used the state average. Part D claims were not adjusted because drugs are commodities with negotiations occurring over pricing between competing drug plans and manufacturers.

Because our spending data covered several years, and Medicare fee schedules increase year to year, we also corrected for changes in prices over time. All claims were set to 2013 U.S. dollars. Inpatient hospitalization claims were adjusted based on the standardized base amount. All hospital outpatient and ambulatory claims were adjusted based on the standard OPPS conversion factor for the calendar year. Physician services, E $+\mathrm{M}$, procedure and imaging claims were adjusted based on Hanh (2014). ${ }^{26}$ SNF, HHA, hospice, long-term care, inpatient psychiatric, and inpatient rehab were adjusted based on the appropriate CMS MarketBasket data. The payment amount for outpatient, carrier, HHA, hospice, and DME claims on or after April 1, 2013, were adjusted to account for sequester. Part D drugs were adjusted using the Consumer Price Index for prescription drugs, and Part B drugs were adjusted using the PPI for pharmaceutical preparations. Clinical labs were adjusted based on the Consumer Price Index for medical care services. Ambulance services were adjusted based on the CMS Ambulance Fee Schedule. DME claims were adjusted based on the MEI

CMS = Centers for Medicare \& Medicaid Services; DME=durable medical equipment; DRG=diagnosis-related group; $E+M=$ evaluation and management; FIPS = Federal Information Processing Standards; HHA = home health agency; ICD-O-3=International Classification of Diseases for Oncology, Third Revision; IRF =inpatient rehabilitation facility; LOS=length of stay; MEI=Medicare Economic Index; NSCLC=non-small cell lung cancer; OPPS = outpatient prospective payment system; PPI = Producer Price Index; RCC = renal cell carcinoma; $S N F=$ skilled nursing facility. 


\section{APPENDIX B Cost per Additional Life-Year}

For the 2 cancer types that showed a statistically significant improvement in survival for a $\$ 10,000$ increase in standardized spending, we calculated the cost per additional life-year as follows.

NSCLC

In general, with proportional hazards the following relationship holds:

$\mathrm{S}_{1}(t)=\mathrm{S}_{0}(t)^{r}$

where, in our context, $r$ is the hazard ratio for a $\$ 10$ thousand increase in standardized spending, $S_{1}(t)$ is survival at time $t$ with $\$ 10$ thousand more of standardized spending, and $S_{0}(t)$ is survival at time $t$ at the base value of standardized spending.

Our method was to calculate the value of $S_{0}(t)$ at the life expectancy of a patient with $\$ 10$ thousand more in spending where, by definition, $S_{1}(t)$ equals 0.5 . We then used the Kaplan-Meier (K-M) curve that we generated from our NSCLC data as the base case survival curve and found that value of $t$ associated with the life expectancy at $\$ 10$ thousand more in spending. We took the difference between that value and the life expectancy of someone with base level spending, which by definition was the value of $t$ at which $S_{0}(t)$ equals 0.5 , which we also read from the K-M curve, to get the additional amount of life expectancy at $\$ 10$ thousand more in standardized episode spending.

Applying the above method:

$S_{0}(t)=(0.5)^{1 / 0.929}=0.4742$.

Reading from the K-M survival curve, the value derived in (2) was associated with 22 months of survival since diagnosis. The difference between that and the value from the K-M curve where $S_{0}$ is 0.5 equaled a 2 -month increase in survival.

Multiplying by 6 to translate to l-year of survival gave $\$ 60$ thousand per episode. One must also account for multiple episodes per patient. After adjusting for 1.787 episodes per patient, we concluded that our survival estimates imply a cost of \$107 thousand per additional life-year in the case of NSCLC.

In order to convert to a cost per QALY measure, we had to adjust for quality of life for an NSCLC patient. There are several estimates in the literature. ${ }^{18-20}$ Nafees et al. (2008) provides a representative range from 0.67 to 0.47 , yielding cost per QALY values ranging from about $\$ 160$ thousand to about $\$ 228$ thousand. 19

MM

Analogous calculations were done to evaluate the additional life expectancy for a $\$ 10$ thousand increase in spending for MM. Using the HR from the Cox regression for MM:

$S_{0}(t)=(0.5)^{1 / 0.866}=0.4491$

From the K-M curve for MM, the value calculated in (3) was associated with 78 months of survival, while $S_{0}(t)$ was 0.5 at 69 months of survival for an increase of 11 months in life expectancy for $\$ 10$ thousand more in standardized spending per episode. Converting to a full-year basis by multiplying by $12 / 11$ and adjusting for 3.267 episodes per patient, our survival estimates imply a cost of $\$ 36$ thousand per additional life-year. Using the quality adjustment for myeloma patients of 0.52 reported in Kharroubi et al. (2015) gave a cost per QALY of $\$ 69$ thousand. ${ }^{21}$

\section{Alternative Method}

An alternative method of calculating the implied cost per additional life-year yields very similar results. If one assumes an exponential distribution rather than using the empirical K-M curve to approximate the survival function, the following relationship holds:

$\mathrm{r}=m_{0} / m_{1}$

(4)

where $m_{0}$ is median survival in the base case and $m_{1}$ is median survival at $\$ 10$ thousand more in standardized spending. Using equation (4) with our NSCLC results and using our K-M curve at survival equal to 0.5 to yield $m_{0}$ equal to 20 months, we obtain

$m_{1}=m_{0} / r={ }^{20 / 0.929}=21.53$ months

or a 1.53-month gain in life expectancy for $\$ 10$ thousand more in spending per episode. Translating to a 12-month gain and correcting for multiple episodes per patient, we obtained $\$ 140$ thousand as the cost of an additional life-year.

Applying equation (4) to the case of MM, analogous calculations show

$m_{1}=m_{0} / r={ }^{69 / 0.866}=79.68$ months

(6)

or a 10.68-month gain in life expectancy for $\$ 10$ thousand more in spending per episode. That translates to $\$ 37$ thousand as the cost of an additional life-year.

We see that both methods yield similar results. We prefer the first method because it uses the K-M curve from the data as the approximation of the survival function and does not require the assumption of an exponentially distributed survival curve.

MM= multiple myeloma; NSCLC=non-small cell lung cancer; QALY=quality-adjusted life-year. 